\title{
Diferencias territoriales en el reconocimiento de la discapacidad de niños y adolescentes con cáncer en España
}

\author{
Territorial differences in recognition of disability of children and \\ adolescents with cancer in Spain
}

\begin{abstract}
Resumen
El impacto del diagnóstico de cáncer en niños y adolescentes afecta a todas las áreas individuales y familiares desde el momento del diagnóstico colocándolos en situación de discapacidad. Existe un conocimiento empírico, no cuantificado, de que el reconocimiento de la discapacidad no es igual en todas las comunidades autónomas. Nos planteamos conocer el estado actual de concesión de la discapacidad de niños y adolescentes con cáncer y su variabilidad entre comunidades autónomas. Se utilizó una muestra de 1288 casos nuevos de niños y adolescentes diagnosticados de cáncer entre 0 y 18 años en el periodo 2014-2016. El 26,9\% solicitó valoración de discapacidad y se concedió en el 92,8\% con amplia variabilidad entre comunidades autónomas. El tiempo medio entre solicitud y diagnóstico es 6 meses, variando entre 1 y 11 meses entre comunidades autónomas. El tiempo medio entre solicitud y concesión es 3,4 meses, variando según comunidades autónomas entre 1 y 5 meses.
\end{abstract}

\section{Palabras clave}

Cáncer pediátrico, desigualdad, integración, sanidad.

\begin{abstract}
The impact of the diagnosis of cancer in children and adolescents affects all individual and family areas since the moment of diagnosis, placing them in a situation of disability. There is an empirical knowledge, not quantified, that the recognition of the disability is not the same in all the autonomous communities. We set out to know the current state of disability grant of children and adolescents with cancer and its variability between autonomous communities. A sample of 1288 new cases of children and adolescents between 0 and 18 years old who were diagnosed with cancer in the $2014-2016$ period was used. $26,9 \%$ requested disability assessment and it was granted in $92,8 \%$ of the cases with wide variability among autonomous communities. The average time between request and diagnosis is 6 months varying between 1 and 11 months between autonomous communities. The average time between application and grant is 3,4 months, varying according to autonomous communities between 1 and 5 months.
\end{abstract}

\section{Keywords}

Pediatric cancer, inequality, integration, health.

1. En representación de los participantes en el estudio de la Comisión de Trabajo Social de las diferentes asociaciones miembro de la FEPNC: Ana Rosa Muniáin (ADANO), María Isidora Cañete, Claudio Jesús Jiménez y Concepción Moreno (AFANION), Juan José Sánchez (AFACMUR), Gemma Caparrós (ALES), Olga Tassara (ANDEX), Raquel Cano (AOEX), Gemma Sevillano (ASPANOA), Nuria Cardona, Ana Herrero, Ana Catalá, Antonio Arteseros (ASPANION), Raquel Centeno (ASION), Pablo Morillas (ARGAR), Isabel Fernández (GALBAN), Alba Ruíz (PEQUEÑO VALIENTE) y María Alonso (PYFANO).

\author{
Jorgina Garrido Casas \\ <sanidad@cancerinfantil.org> \\ Federación Española de Padres de \\ Niños con Cáncer (FEPNC). España
}

\section{Carmen Menéndez Llaneza}

<carmenmll@cancerinfantil.org>

Federación Española de Padres de Niños con Cáncer (FEPNC). España

\section{Patricia Pico Gómez-Pavón} $<$ federacipn@cancerinfantil.org>

Federación Española de Padres de Niños con Cáncer (FEPNC). España

Miguel Ángel de la Cal López <mcal@delacalmoreno.com>

Federación Española de Padres de Niños con Cáncer (FEPNC). España

\section{Silvia Bozalongo Jalón-} Mendiri ${ }^{1}$

<trabajadorasocial@menores concancer.org>

Asociación Riojana de Familiares y Amigos de Niños con Cáncer (FARO). España

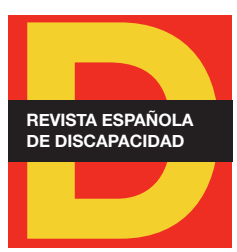

Para citar:

Garrido, J. et al. (2019): "Diferencias territoriales en el reconocimiento de la discapacidad de niños y adolescentes con cáncer en España”. Revista Española de Discapacidad, 7 (I): 49-66

Doi: <https://doi.org/10.5569/23405104.07.01.03>

Fecha de recepción: 04-04-2018 Fecha de aceptación: 10-05-2019 


\section{Introducción}

La Organización Mundial de la Salud en su Clasificación Internacional del Funcionamiento de la Discapacidad y de la Salud concibe el funcionamiento y discapacidad de una persona como una "interacción dinámica entre los estados de salud (enfermedades, trastornos, lesiones, traumas, etc.) y los factores contextuales". Incluyendo en los componentes de funcionamiento y discapacidad tanto aspectos problemáticos (deficiencias, limitaciones en la actividad o restricciones en la participación) como no problemáticos (ej. neutrales) de la salud o "relacionados con la salud" (OMS, 2001: 9). El diagnóstico de cáncer afecta a todas las áreas tanto individuales como familiares de los niños y adolescentes desde el momento del diagnóstico, poniendo a este colectivo en una situación de discapacidad.

Desde el primer día de tratamiento se interrumpen todas las actividades de su vida diaria como ir al colegio, jugar, relacionarse con otros niños, compartir ocio..., y en la mayoría de los casos este periodo puede alargarse 1 año o más. Esta discapacidad es frecuentemente temporal hasta la finalización del tratamiento, que suele ser agresivo y prolongado. En este momento son ya más evidentes las alteraciones crónicas de salud. Aunque la supervivencia del cáncer infantil en España alcanza, a los 5 años, un $79 \%$ (Peris Bonet et al., 2018), en un estudio realizado por Oetfinger et al. en 2006, con una muestra de 10.937 supervivientes de cáncer infantil, un $73,4 \%$ presentaba una condición crónica de salud a los 30 años del diagnóstico de cáncer y de estos en un $42,4 \%$ era grave, discapacitante o amenazante para la vida. Por tanto, conviene resaltar que la discapacidad de los niños y adolescentes con cáncer es de dos tipos: una tras el diagnóstico, que es universal y frecuentemente temporal, y otra posterior que suele ser secundaria al tipo de tumor, a los efectos adversos de los tratamientos recibidos y a la situación traumática vivida por los supervivientes (Barrera et al., 2005: 1751; Hjern et al., 2007: 5262; Hudson et al., 2013: 2371; Armstrong et al., 2014: 1218).

La normativa estatal actual (RD 1971/1999; RD 1856/2009; RD 1364/2012 y RD 1/2013) no contempla la situación de discapacidad en la que se encuentra el niño/adolescente al ser diagnosticado y comenzar cualquiera de los tratamientos agresivos y prolongados que se le van a proporcionar. En el RD 1971/1999 de 23 de diciembre se establece que:

- Los enfermos neoplásicos sometidos a tratamientos potencialmente curativos deberán ser evaluados una vez finalizados los mismos.

- En los casos de tratamiento quirúrgico aislado, el grado de discapacidad será evaluado transcurridos seis meses desde la intervención.

- Durante el periodo de aplicación de tratamientos poliquimioterápicos y radioterápicos, se mantendrá la valoración de la discapacidad que previamente tuviera el enfermo si la hubiere.

- En el caso de enfermos sometidos a trasplante de médula ósea, la valoración, si la hubiere, se mantendrá hasta 6 meses después de realizado el trasplante, procediéndose entonces a su reevaluación.

Esperar 6 meses o a la finalización del tratamiento no se ajusta a las necesidades de este colectivo y priva a los niños y a sus familiares del acceso a los servicios y prestaciones a los que tienen derecho (ayuda a domicilio, ayudas para rehabilitación y asistencia sanitaria y farmacéutica, subsidio de movilidad y/o compensación de gastos de transporte, prestación económica a la familia por hijo a cargo hasta los 18 años, etc.). Se ha estimado 
por las asociaciones federadas que el presupuesto medio que los familiares tienen que dedicar a la atención de su hijo enfermo oscila entre $400 €$ y $600 €$ mensuales. Este incremento del gasto familiar comienza en el momento del diagnóstico del niño o adolescente. El soporte recibido por estos niños y sus familias, p.ej. psicológico, educativo y de servicios sociales, favorece la capacidad de integración con otros niños y adolescentes de su edad y puede favorecer su integración socio-laboral en la edad adulta (Barrera et al., 2005: 1751).

La información sobre los niños y adolescentes con cáncer se ha centrado en nuestro país, como en otros países de nuestro entono, en conocer la incidencia de tumores y su supervivencia. Existe, por tanto, un déficit de información por parte de las autoridades sanitarias respecto a la situación disfuncional de los menores durante el periodo de tratamiento, que no se refleja en los criterios de aplicación de las normas generales del RD 1971/1999, de 23 de diciembre.

La información aportada por diferentes asociaciones federadas y colaboradoras ${ }^{2}$ de la Federación Española de Padres de Niños con Cáncer (FEPNC) muestra que las solicitudes de valoración, en línea con la normativa, son en general bajas pero con amplias variaciones entre comunidades autónomas. Las diferencias entre comunidades autónomas no solo se observan en el número de solicitudes sino en los espacios de tiempo desde el diagnóstico hasta la solicitud y desde la solicitud hasta la resolución. Este hecho hace pensar que, a pesar de los baremos de discapacidad, en los que no se recoge de forma explícita la concesión de discapacidad a niños con cáncer desde el diagnóstico, se están tomando decisiones diferentes en los distintos centros de valoración. Diferencias que se producen no únicamente entre comunidades autónomas sino dentro de las propias comunidades, como nos indican diferentes trabajadoras sociales de algunas de las asociaciones.

Con este proyecto se decidió estudiar la situación actual de los niños/adolescentes con cáncer respecto a la valoración y concesión de discapacidad. La confirmación de la información aportada por las asociaciones de la FEPNC permitirá poder solicitar, con información basada en la evidencia, la homogeneidad de criterios de valoración en todo el territorio nacional y así asegurar que todas las familias, independientemente de su lugar de residencia, tengan acceso, desde el momento del diagnóstico, a una ayuda social que facilite una atención integral real, física, psíquica y social, al hacerles beneficiarios de los servicios sociales que necesitan.

\section{Objetivos}

El objetivo general del proyecto DISCAIN, en el que se enmarca este estudio, es conocer el estado actual de concesión de discapacidad a los niños y adolescentes con cáncer ( 0 a 19 años), ya sea a consecuencia de la enfermedad o de su tratamiento y su variabilidad entre comunidades autónomas.

Específicamente nos planteamos:

2. ADANO (Navarra), AFACMUR (Región de Murcia), AFANION (Castilla-La Mancha), AFANOC (Cataluña), ALES (Andalucía-Jaén), ANDEX (Andalucía-Sevilla), AOEX (Extremadura), ARGAR (Andalucía-Almería), ASANOG (Galicia), ASION (Comunidad de Madrid), ASPANION (Comunidad Valenciana), ASPANOA (Aragón), E. VILLAVECHIA (Cataluña), FARO (La Rioja), GALBAN (Asturias), PEQUEÑO VALIENTE (Canarias), PYFANO (Castilla y León). 
1. Conocer la variación entre comunidades autónomas respecto a:

a. Solicitudes y concesiones de discapacidad de niños con cáncer.

b. Tiempo transcurrido entre el diagnóstico y la solicitud de discapacidad.

c. Tiempo transcurrido entre la solicitud y la concesión de discapacidad.

2. Identificar diferencias sociodemográficas y sanitarias entre los menores con cáncer con un grado de discapacidad reconocido y aquellos que no solicitan valoración de discapacidad o les ha sido denegada.

\section{Metodología}

\section{Procedimiento}

Para recoger información se solicitó la participación de las asociaciones de la FEPNC. Decidieron participar 14 asociaciones: ADANO, AFACMUR, AFANION, ALES, ANDEX, AOEX, ARGAR, ASION, ASPANION, ASPANOA, FARO, GALBAN, PEQUEÑO VALIENTE y PYFANO. Distribuidas en 12 comunidades autónomas: Andalucía, Aragón, Principado de Asturias, Canarias, Castilla-La Mancha, Castilla y León, Comunidad de Madrid, Comunidad Valenciana, Extremadura, Navarra, Región de Murcia y La Rioja. Las trabajadoras sociales, una vez firmado el acuerdo de confidencialidad, se comprometieron a introducir los datos de los niños/ adolescentes cuyos padres dieron el consentimiento para intervenir en el proyecto.

Desde el 1 de enero de 2014 hasta el 31 de diciembre de 2016 se fueron introduciendo nuevos casos (108 se introdujeron a lo largo de 2017) y actualizando los datos de los ya existentes (nuevos diagnósticos, nuevos tratamientos, situación sanitaria, discapacidad).

\section{Instrumento}

Este estudio es una explotación parcial de datos del proyecto DISCAIN iniciado en 2013 por la FEPNC en colaboración con el Instituto de Salud Carlos III y financiado por el Real Patronato sobre Discapacidad. Ese año se determinaron las variables de análisis relevantes para los objetivos, así como el diseño de una base de datos informática para el registro de la información. Se elaboró una base de datos en la que como principales variables se encuentran las que aparecen en la tabla 1.

Tabla 1. Definición de variables
\begin{tabular}{|l|l|l|}
\hline Sociodemográficas & Sanitarias & Discapacidad \\
\hline Sexo & Diagnóstico & Solicitud de discapacidad \\
\hline Edad & Fecha de diagnóstico & Fecha de solicitud \\
\hline Fecha de nacimiento & Edad primer diagnóstico & Fecha de concesión \\
\hline Comunidades autónomasde residencia & Tratamiento & Grado de discapacidad concedido \\
\hline & Situación sanitaria & Tiempo transcurrido diagnóstico-solicitud \\
\hline & & Tiempo transcurrido solicitud-concesión \\
\hline
\end{tabular}

Fuente: elaboración propia. 
Las categorías de las variables: sexo, edad, comunidad autónoma de residencia, diagnóstico, tratamiento y situación sanitaria aparecen en la descripción de la muestra en la tablas 2 y 3 . Las categorías de grado de discapacidad concedido son: 0-32\%; $33 \%-64 \%$; >= 65\%; Ninguna. Y de tiempo transcurrido diagnóstico-solicitud y solicitud concesión son: <= 1 mes; >1 - <= a 2 meses, > 2 - <= 3 meses, > 3 - <= a 6 meses; > 6 meses.

\section{Muestra}

Está constituida por un total de 1288 casos nuevos que se seguirán durante un periodo de 5 años. Como se puede observar en la tabla 2, hay una mayor proporción de niños (55\%) que de niñas (45\%), las edades que prevalecen son entre 0 y 5 años $(46,4 \%)$ y residen fundamentalmente en la Comunidad de Madrid $(21,8 \%)$, Comunidad Valenciana $(17,0 \%)$ y Andalucía $(11,2 \%)$

\begin{tabular}{|c|c|c|}
\hline Características sociodemográficas & Participantes & $\%$ \\
\hline Sexo & \multicolumn{2}{|l|}{1288} \\
\hline $\begin{array}{l}\text { Mujer } \\
\text { Hombre }\end{array}$ & $\begin{array}{l}580 \\
708 \\
\end{array}$ & $\begin{array}{l}45,0 \\
55,0 \\
\end{array}$ \\
\hline Grupos de Edad (años primer diagnóstico) & \multicolumn{2}{|l|}{1288} \\
\hline$\leq 2$ & 317 & 24,6 \\
\hline$\geq 3-\leq 5$ & 280 & 21,7 \\
\hline$\geq 6-\leq 8$ & 199 & 15,5 \\
\hline$\geq 9-\leq 11$ & 184 & 14,3 \\
\hline$\leq 12-\leq 14$ & 198 & 15,4 \\
\hline$\geq 15$ & 110 & 8,5 \\
\hline Comunidad autónoma de residencia & \multicolumn{2}{|l|}{1288} \\
\hline Andalucía & 144 & 11,2 \\
\hline Aragón & 71 & 5,5 \\
\hline Asturias, Principado de & 51 & 4,0 \\
\hline Baleares & 1 & 0,1 \\
\hline Canarias & 102 & 7,9 \\
\hline Cantabria & 2 & 0,2 \\
\hline Castilla-La Mancha & 129 & 10,0 \\
\hline Castilla y León & 77 & 6,0 \\
\hline Cataluña & 0 & 0,0 \\
\hline Comunidad Valenciana & 219 & 17,0 \\
\hline Extremadura & 33 & 2,6 \\
\hline Galicia & 5 & 0,4 \\
\hline Madrid, Comunidad de & 281 & 21,8 \\
\hline Murcia, Región de & 90 & 7,0 \\
\hline Navarra, Comunidad Foral de & 48 & 3,7 \\
\hline País Vasco & 11 & 0,9 \\
\hline Rioja, La & 24 & 1,9 \\
\hline
\end{tabular}

Fuente: elaboración propia. 
Atendiendo a su situación sanitaria (tabla 3) los diagnósticos de "Leucemias, enfermedades mieloproliferativa y mielodisplásicas" (33,5\%), "SNC y miscelánea de neoplasias intracraneales e intraespinales" (17,9\%) y "Linfomas y neoplasias reticuloendoteliales" (12,6\%) son los que aparecen más representados, constituyendo el $64,0 \%$ del total de la muestra. El tratamiento que han recibido con mayor frecuencia es la quimioterapia $(88,3 \%)$ seguido de la cirugía $(29,9 \%)$ y la radioterapia $(14,6 \%)$. El $42,5 \%$ se encuentran en tratamiento.

\section{Tabla 3. Características sanitarias de la muestra}

\begin{tabular}{|c|c|c|}
\hline Características sanitarias & Participantes & $\%$ \\
\hline Grupo diagnóstico & \multicolumn{2}{|l|}{1288} \\
\hline Leucemias, enfermedades mieloproliferativas y mielodisplásicas & 432 & 33,54 \\
\hline SNC y miscelánea de neoplasias intracraneales e intraespinales & 231 & 17,9 \\
\hline Linfomas y neoplasias reticuloendoteliales & 162 & 12,6 \\
\hline Tumores óseos & 121 & 9,4 \\
\hline Neuroblastomas y otros tumores de células nerviosas periféricas & 78 & 6,1 \\
\hline Sarcomas de tejidos blandos y otros extraóseos & 52 & 4,0 \\
\hline Enfermedades inmunohematológicas & 51 & 4,0 \\
\hline Tumores renales & 43 & 3,3 \\
\hline Retinoblastomas & 37 & 2,9 \\
\hline Tumores de células germinales, tumores trofoblásticos y neoplasias gonadales & 23 & 1,8 \\
\hline Tumores hepáticos & 22 & 1,7 \\
\hline Melanomas malignos y otras neoplasias epiteliales malignas & 5 & 0,4 \\
\hline Otras neoplasias malignas y no especificadas & 31 & 2,4 \\
\hline \multicolumn{3}{|l|}{ Tratamiento } \\
\hline Quimioterapia & 910 & 88,3 \\
\hline Radioterapia & 150 & 14,6 \\
\hline Cirugía & 308 & 29,9 \\
\hline Trasplante & 107 & 10,4 \\
\hline Rehabilitación & 20 & 1,9 \\
\hline Tomoterapia & 18 & 1,7 \\
\hline Ensayos clínicos & 10 & 1,0 \\
\hline Otros & 24 & 2,3 \\
\hline Total de tratamientos recibidos (un mismo caso ha podido recibir varios tratamientos) & 1547 & \\
\hline Total de casos & 1030 & 100 \\
\hline Perdidos de sistema & 258 & \\
\hline Total & 1288 & \\
\hline \multicolumn{3}{|l|}{ Situación sanitaria } \\
\hline Alta & 8 & 0,8 \\
\hline Revisión menor a 3 meses & 269 & 25,8 \\
\hline Revisión entre 3 y 6 meses & 130 & 12,5 \\
\hline Revisión mayor a 6 meses & 29 & 2,8 \\
\hline Revisión anual & 6 & 0,6 \\
\hline En tratamiento & 443 & 42,5 \\
\hline Fallecido & 157 & 15,1 \\
\hline Total & 1042 & 100 \\
\hline Perdidos sistema & 246 & \\
\hline Total & 1288 & \\
\hline
\end{tabular}

Fuente: elaboración propia. 


\section{Análisis estadístico}

Se utilizaron como medidas de distribución de variables, según el tipo de variable (nominal o de intervalo): la frecuencia, media, mediana y desviación típica.

Para analizar las diferencias sociodemográficas (sexo, edad, comunidad autónoma) y sanitarias (diagnóstico, tratamiento, situación sociosanitaria) entre niños con cáncer con discapacidad (grado de discapacidad reconocido) y no discapacidad (no solicitada o no concedida) se utilizó el estadístico chi-cuadrado.

En los casos en que no se cumplían los requisitos para aplicar chi-cuadrado se utilizó el análisis de los residuos ajustados tipificados que nos permitieron determinar la asociación entre los valores de cada variable: discapacidad y cada comunidad autónoma, tratamiento recibido y diagnóstico asignado y no discapacidad y cada comunidad autónoma, tratamiento recibido y diagnóstico asignado.

En todos los análisis se utilizó el programa estadístico SPSS 20.

\section{Resultados}

Solicitudes de discapacidad

Se solicitó una valoración de discapacidad en el $26,9 \%$ de los casos con una amplia variabilidad entre comunidades autónomas. Las comunidades de Principado de Asturias (56,9\%), Comunidad Valenciana $(56,2 \%)$ y Región de Murcia $(41,1 \%)$ son en las que más valoraciones se solicitan.

Por el contrario, son las comunidades de Andalucía (7,6\%) Aragón (11,3\%), Comunidad Foral de Navarra $(12,5 \%)$ y La Rioja $(12,5 \%)$, en las que menos solicitudes se realizan (ver figura 1$)$.

El tiempo medio entre el primer diagnóstico y la solicitud de valoración es de seis meses (Media: 6,40 meses, dt: 6,632) con un rango de variación de medias entre Comunidades autónomas entre uno y once meses (con la excepción de La Rioja que con tres solicitudes presenta una media de 28 meses) (ver figura 2).

Las comunidades autónomas con menor media de tiempo entre el diagnóstico y la solicitud de valoración son: Principado de Asturias $(n=28)$, Canarias $(n=22)$, Castilla y León $(n=15)$ y Comunidad de Madrid $(n=$ 22). En todas ellas se observa una asociación estadísticamente significativa con la solicitud de discapacidad al mes o menos del diagnóstico $(p<0,05)$. En estas comunidades el $40 \%$ de los casos o más solicita la discapacidad dentro del primer mes desde que fueron diagnosticados.

Por el contrario, es en la Comunidad Valenciana $(n=106)$, Comunidad Foral de Navarra $(n=6)$ y La Rioja $(n=$ 3 ) donde se dilata más el tiempo de solicitud. El $100 \%$ de las solicitudes de valoración en La Rioja $(p<0,05)$, el $83,3 \%$ en la Comunidad Foral de Navarra $(p<0,05)$ y el $44,3 \%$ en Comunidad Valenciana $(p<0,05)$ se realizan a partir a partir de los 6 meses del diagnóstico, como está establecido en la ley (1971/1999 de 23 de diciembre). 
Figura 1. Distribución de solicitudes de valoración de discapacidad según comunidad autónoma de residencia. Porcentajes

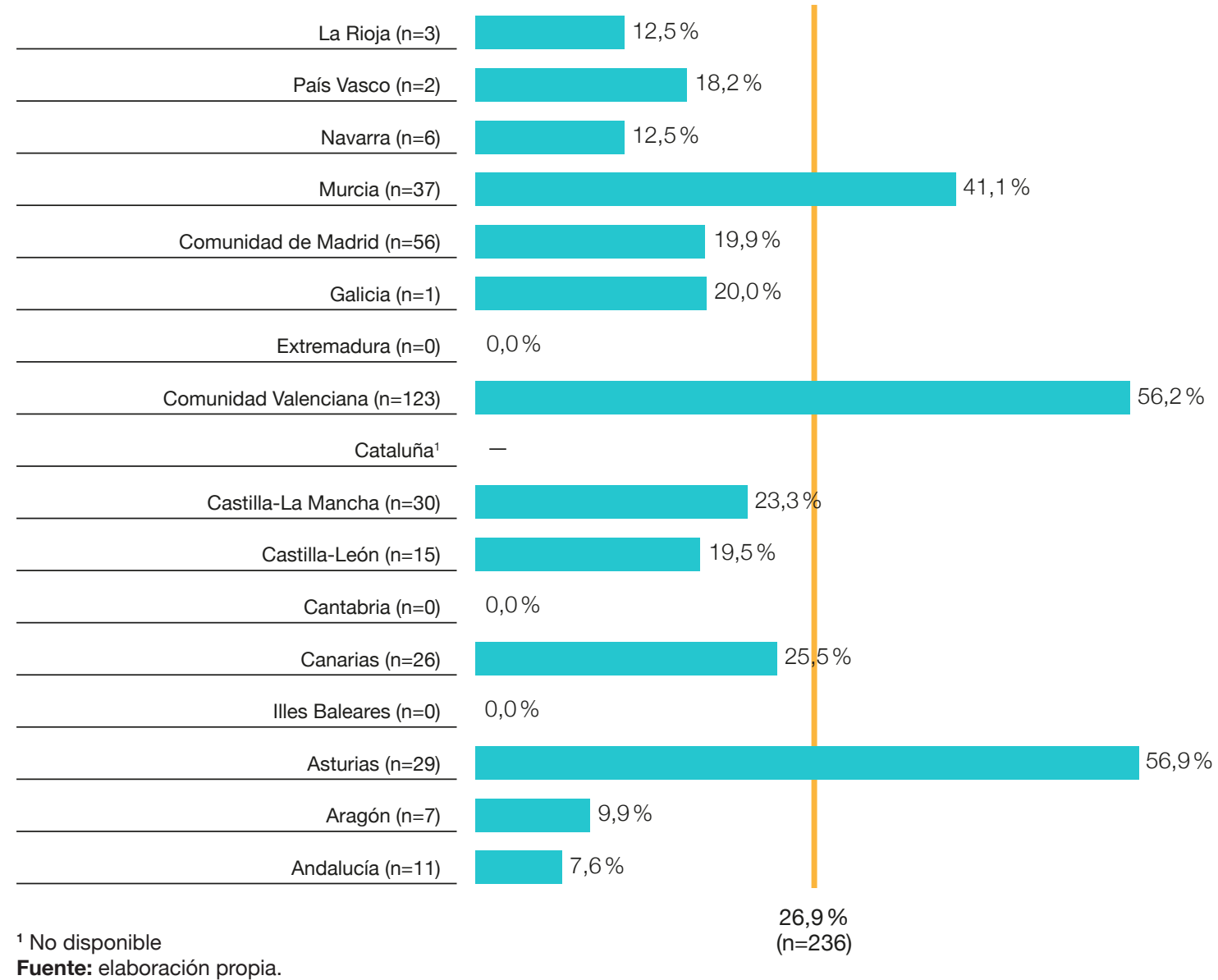

Figura 2. Media de tiempo y desviación típica (dt) entre el primer diagnóstico y la solicitud de discapacidad según la comunidad autónoma de residencia. Barra horizontal: media y desviación típica nacional

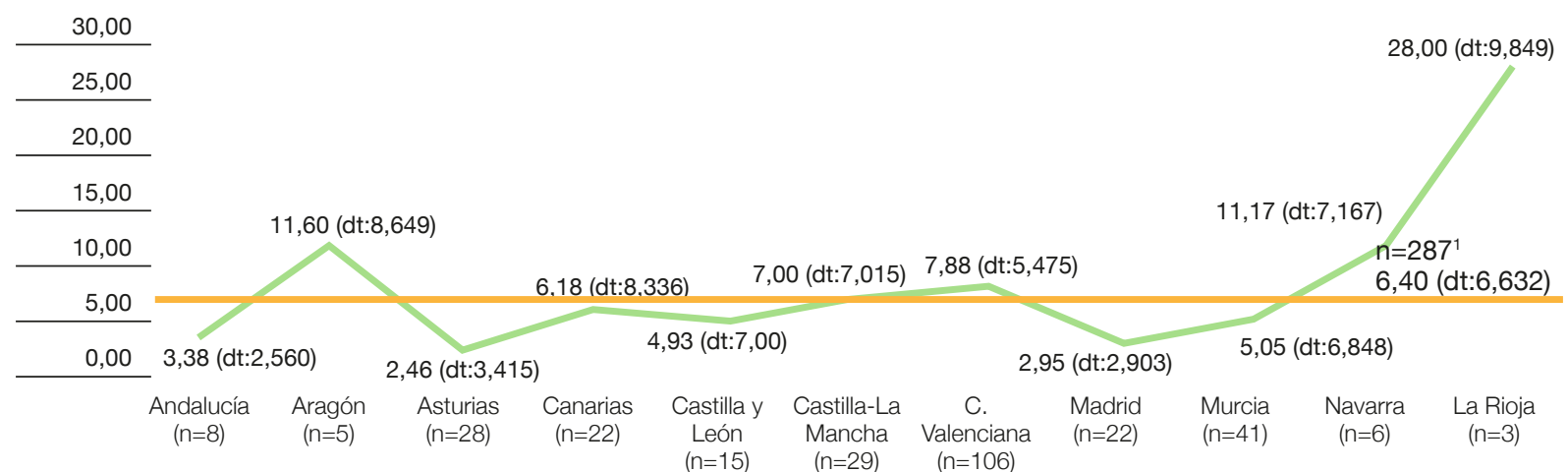

${ }^{1}$ No se han incluido en el gráfico las comunidades autónomas de: Galicia y País Vasco por tener 2 casos o menos de solicitud; Baleares, Cantabria y Extremadura, por no tener ningún caso de solicitud de discapacidad; Cataluña, no se dispone de información. Fuente: elaboración propia. 


\section{Concesiones de discapacidad}

Se conceden el 92,8\% de las solicitudes de discapacidad. Destacan las comunidades autónomas de Andalucía y Canarias en las que se concedió la discapacidad en el $100 \%$ de los casos solicitados. En torno al $100 \%$ también se encuentran la Comunidad de Madrid (98,2\%) y la Región de Murcia (97,3\%).

Los niveles más bajos de concesiones se encuentran la Comunidad Foral de Navarra (33,3\%), País Vasco (50\%) y La Rioja $(66,7 \%)$ (ver figura 3$)$.

De igual forma que en el caso anterior, el cálculo del tiempo medio entre solicitud y concesión de discapacidad se ha realizado con los casos de los que se dispone de información de fecha de solicitud y fecha de discapacidad: 186. No aparecen reflejadas en la figura 4 las comunidades autónomas de las que no se dispone de casos, de alguna de las fechas o únicamente en un caso se ha realizado la solicitud, esto es: Baleares, Cantabria, Cataluña, Extremadura, Galicia y País Vasco.

Figura 3. Distribución de concesiones de discapacidad respecto a solicitudes realizadas, según comunidad autónoma de residencia. Porcentajes

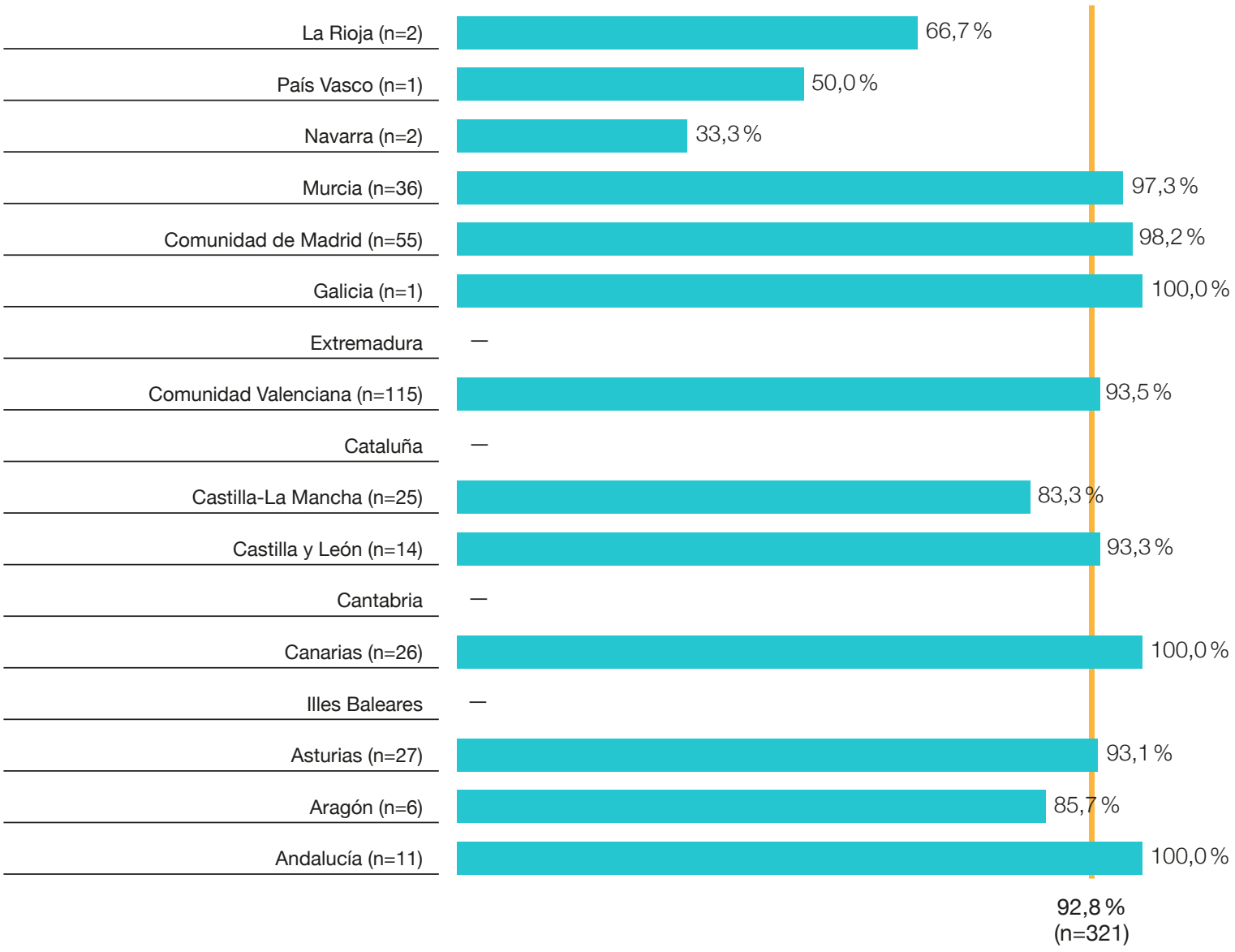

Fuente: elaboración propia. 
El tiempo medio entre la solicitud de valoración y la resolución se encuentra en torno a 3 meses y medio (media: 3,38 meses, dt: 4,122) con un rango de variación de medias entre 1 y 5 meses $(4,94)$, entre comunidades autónomas (ver figura 4). Se encuentran asociaciones estadísticamente significativas entre las comunidades de Andalucía $(p<0,05)$ y la Comunidad Valenciana $(p<0,05)$ y la resolución en un tiempo de 1 mes o menos. El $83 \%$ de los casos que solicitan valoración en Andalucía $(n=6)$ y el $84,4 \%$ de los casos en la Comunidad Valenciana $(n=32)$ se resuelven en menos de 1 mes.

Por el contrario, las comunidades autónomas de la Región de Murcia y la Comunidad Foral de Navarra son en las que más se tarda. En las dos, en torno al $65 \%$ de las solicitudes se resuelven pasados 4 meses. En la Región de Murcia, en concreto, el $28,6 \%$ de las solicitudes se resuelven a los 6 meses o más $(p<0,05)$ (ver figura 4).

Figura 4. Media y desviación típica de tiempo entre la solicitud y la resolución de discapacidad según la comunidad autónoma de residencia. Barra horizontal: media y desviación típica nacional

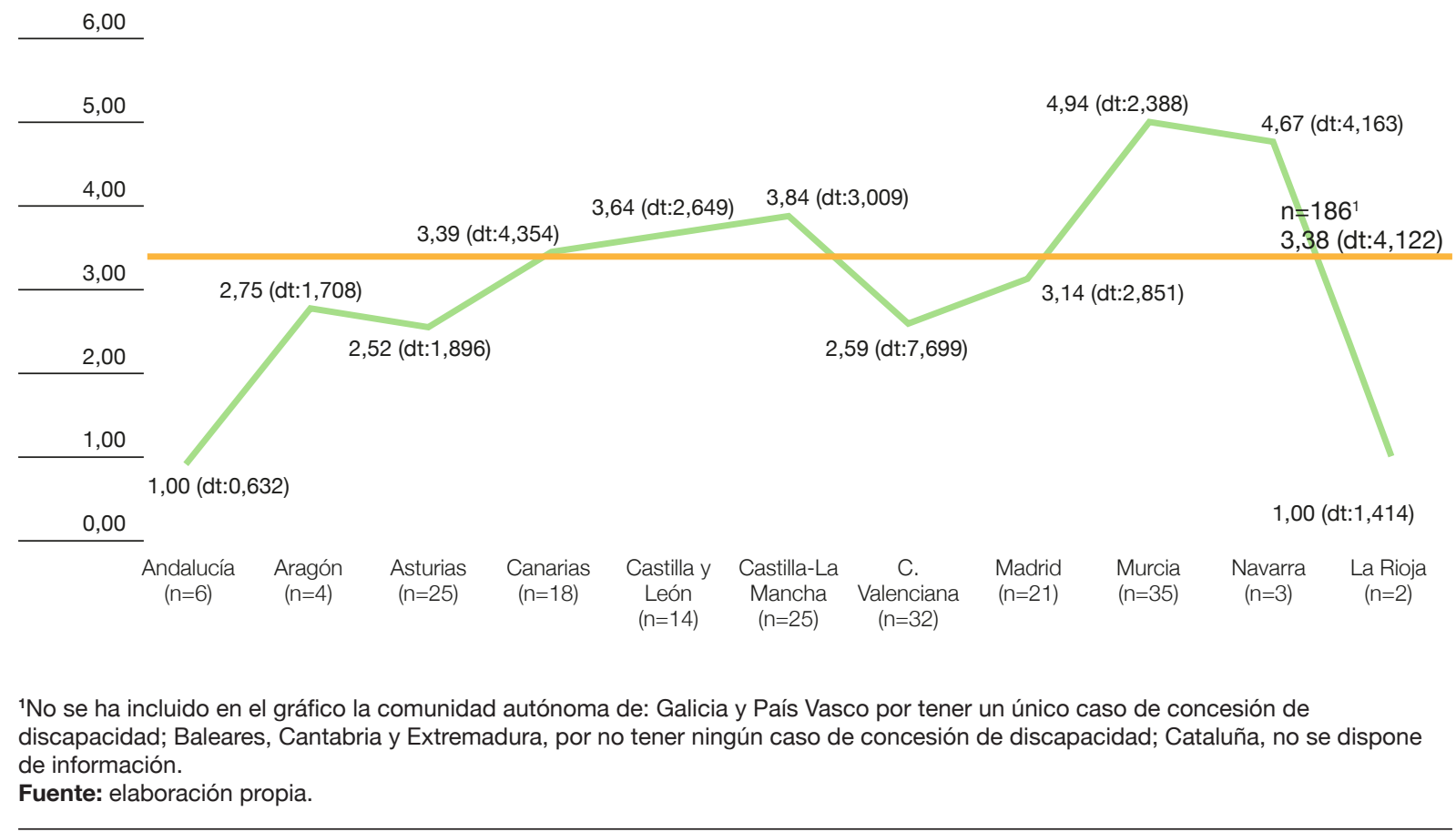

El grado de discapacidad habitualmente concedido se encuentra en el intervalo del 33\%-64\% (57,5\%). Se encuentran asociaciones estadísticamente significativas entre las categorías de Comunidad Valenciana y un grado de discapacidad $>=65 \%(p<0,05)$ y entre Canarias $(p<0,05)$, la Comunidad de Madrid $(p<0,05)$ y la Región de Murcia $(p<0,05)$ con el intervalo de discapacidad de $33 \%-64 \%$ (ver tabla 4). 


\begin{tabular}{|c|c|c|c|c|c|}
\hline \multirow{2}{*}{ Comunidades autónomas } & \multicolumn{4}{|c|}{ Grado de discapacidad } & \multirow{2}{*}{ TOTAL } \\
\hline & $0-32 \%$ & $33 \%-64 \%$ & $>=65 \%$ & Ninguna & \\
\hline $\begin{array}{l}\text { Andalucía } \\
\text { Aragón } \\
\text { Asturias, Principado de } \\
\text { Canarias } \\
\text { Castilla y León } \\
\text { Castilla-La Mancha } \\
\text { Comunidad Valenciana } \\
\text { Galicia } \\
\text { Madrid, Comunidad de } \\
\text { Murcia, Región de } \\
\text { Navarra, Comunidad Foral de } \\
\text { País Vasco } \\
\text { La Rioja }\end{array}$ & $\begin{array}{c}18,2 \\
0 \\
6,9 \\
0 \\
33,3 \\
6,7 \\
3,3 \\
0 \\
1,8 \\
0 \\
0 \\
0 \\
0\end{array}$ & $\begin{array}{l}72,7 \\
85,7 \\
62,1 \\
92,3 \\
46,7 \\
66,7 \\
31,7 \\
100 \\
80.4 \\
75,7 \\
16,7 \\
50 \\
33,3\end{array}$ & $\begin{array}{c}9,1 \\
0 \\
24,1 \\
7,7 \\
13,3 \\
10 \\
58,5 \\
0 \\
16,1 \\
21,6 \\
16,7 \\
0 \\
33,3\end{array}$ & $\begin{array}{c}0 \\
14,3 \\
6,9 \\
0 \\
6,7 \\
16,7 \\
6,5 \\
0 \\
1,8 \\
2,7 \\
66,7 \\
50 \\
33,3\end{array}$ & $\begin{array}{c}11 \\
7 \\
29 \\
26 \\
15 \\
30 \\
123 \\
1 \\
56 \\
37 \\
6 \\
2 \\
3\end{array}$ \\
\hline Total & 4,6 & 57,5 & 30,6 & 7,2 & 346 \\
\hline
\end{tabular}

Fuente: elaboración propia.

Asociación entre discapacidad concedida y no discapacidad (no concedida o no solicitada) según variables sociodemográficas y sanitarias

Se identifican a continuación las asociaciones significativas entre variables sociodemográficas (sexo, edad, comunidad autónoma) y sanitarias (primer diagnóstico, primer tratamiento recibido) y la concesión o no de discapacidad. En ningún caso se cumplen los requisitos para utilizar el estadístico chi-cuadrado por lo que se realizó un análisis de los residuos para determinar las asociaciones entre los valores de las variables en estudio.

La concesión de discapacidad en niños/adolescentes con cáncer es independiente del sexo y la edad de los menores. Sí se encuentran asociaciones estadísticamente significativas entre la categoría de discapacidad concedida y las comunidades autónomas de Principado de Asturias $(p<0,05)$, Comunidad Valenciana $(p<0,05)$ y la Región de Murcia $(p<0,05)$ (ver figura 5$)$. 
Figura 5. Distribución de casos con discapacidad y no discapacidad según comunidad autónoma de residencia (\%). No discapacidad, $\mathrm{n}=$ 981; Discapacidad, $\mathrm{n}=307$

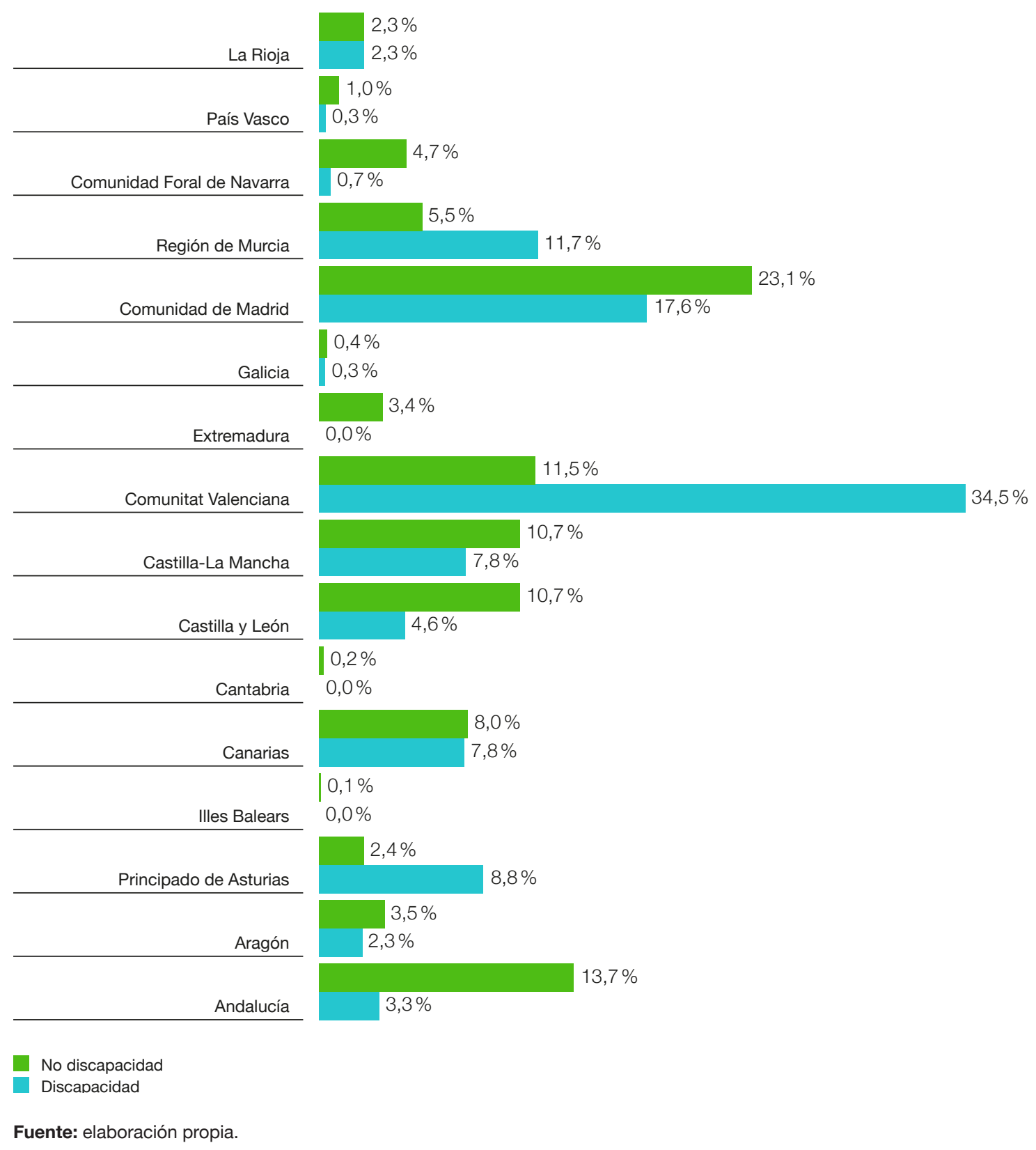

Si tenemos en cuenta las variables sanitarias, se observa una asociación significativa entre la discapacidad y el diagnóstico de "Leucemia" $(p<0,05)$ (ver figura 6). 


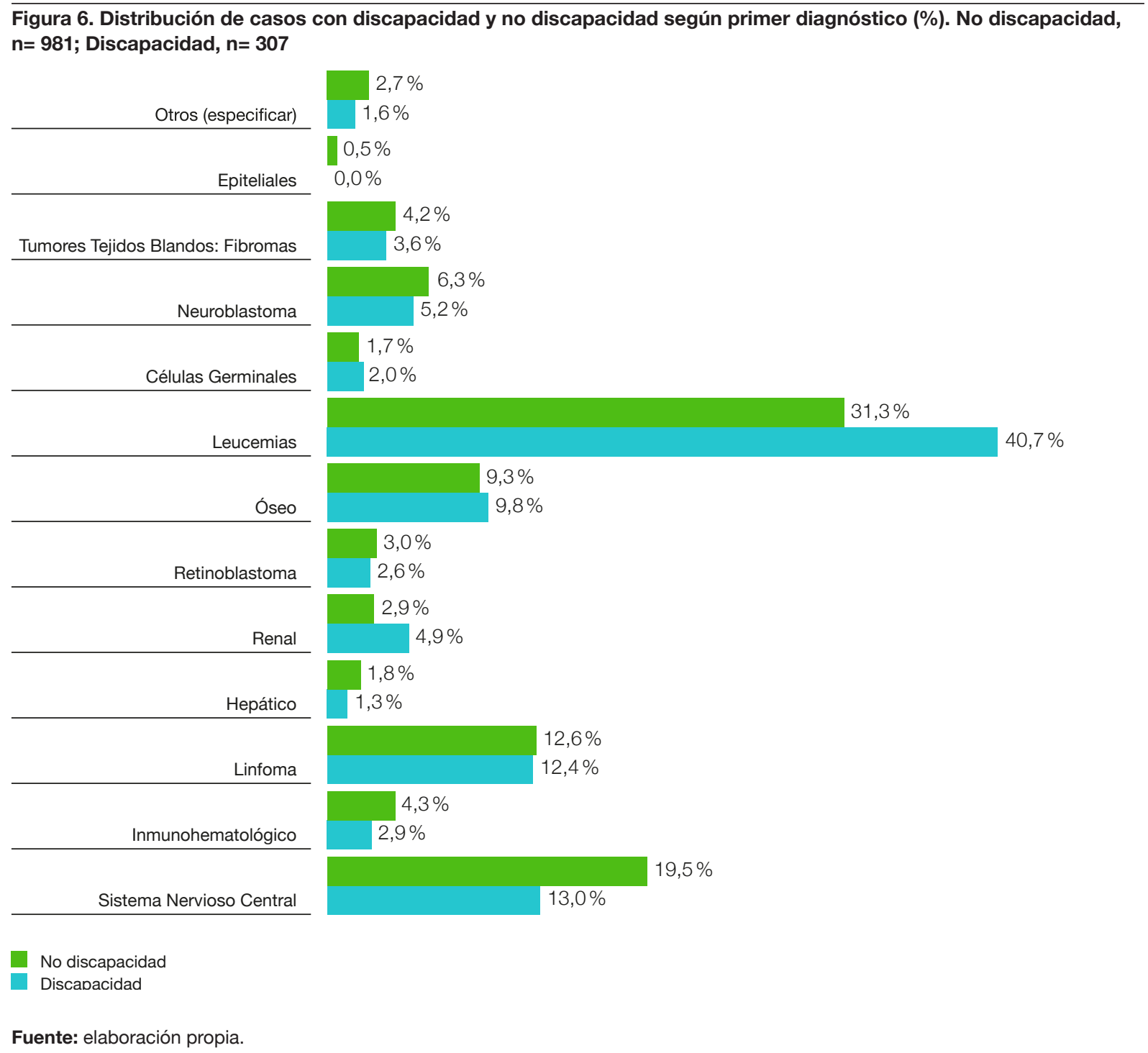

En función del tratamiento encontramos una asociación estadísticamente significativa entre las categorías de discapacidad y quimioterapia $(p<0,05)$ y no discapacidad y cirugía $(p<0,05)$, como primeros tratamientos (ver figura 7). 
Figura 7. Distribución de casos de discapacidad y no discapacidad según primer tratamiento (\%). No discapacidad, $n=981$; Discapacidad, $n=307$
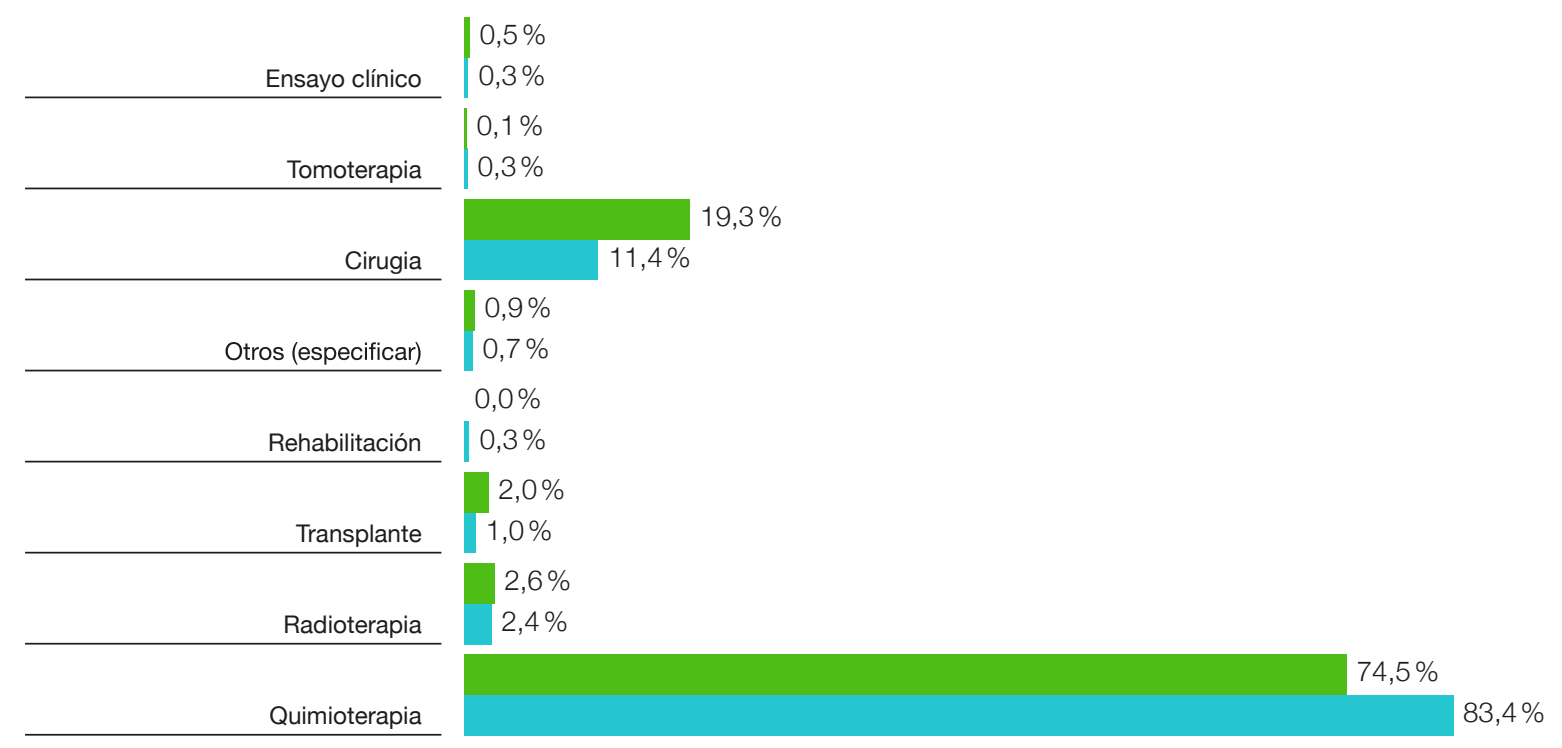

Fuente: elaboración propia.

La asociación entre discapacidad y supervivencia se ha valorado analizando la distribución de la concesión de discapacidad entre los supervivientes y fallecidos y la no discapacidad (no solicitada o no concedida) entre ambos grupos.

Se observa una asociación estadísticamente significativa entre discapacidad y supervivencia (chi-cuadrado= 28,588; $p<0,001$ ) (ver figura 8).

Figura 8. Distribución de concesión de discapacidad según supervivencia (\%). No discapacidad, n=744; Discapacidad, n= 298

$\begin{array}{r}100 \\ \hline 80 \\ \hline 60 \\ \hline 40 \\ \hline 20 \\ \hline 0 \\ \hline\end{array}$

No discapacidad

Discapacidad

Fuente: elaboración propia.

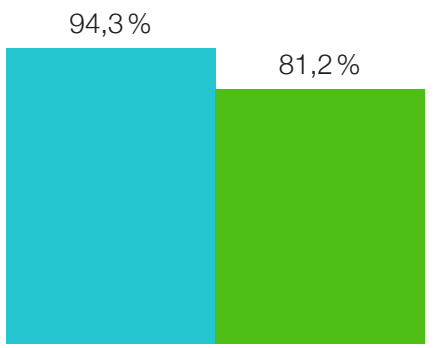

Supervivientes
$18,8 \%$

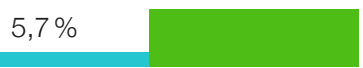

Fallecidos 


\section{Discusión y conclusiones}

Se ha solicitado algún grado de discapacidad a partir del diagnóstico en el $26,9 \%$ de los casos registrados y se ha concedido en el 92,8\% como promedio, existiendo una amplia variabilidad dependiendo de las comunidades autónomas.

Los intervalos de tiempo transcurridos entre el diagnóstico y la solicitud de valoración y entre la solicitud de valoración y la comunicación de la resolución también son muy variables entre comunidades autónomas. El tiempo medio entre el primer diagnóstico y la solicitud de valoración es de 6 meses (media: 6,40 meses, dt: 6,632 ) con un rango de variación de medias entre comunidades autónomas entre 1 y 11 meses. Y el tiempo medio entre la solicitud de valoración y la resolución se encuentra en torno a 3 meses y medio (media: 3,38 meses, dt: 4,122) con un rango de variación entre 1 y 5 meses entre comunidades autónomas. Tiempos máximos, en ambos casos que sobrepasan ampliamente un periodo razonable.

Se confirma el hecho de que los niños y adolescentes con cáncer que solicitan la valoración de discapacidad varía ampliamente entre comunidades autónomas, que son valorados en tiempos diferentes dependiendo de su lugar de residencia y que existe un retraso indeseable en solicitar y resolver la valoración en algunas comunidades autónomas.

Disponer de un grado de discapacidad, solicitada a consecuencia del diagnóstico de cáncer, se encuentra asociado a las comunidades autónomas de Principado de Asturias, Comunidad Valenciana y Región de Murcia, a un primer diagnóstico de leucemia, un primer tratamiento de quimioterapia y ser superviviente. Sin embargo, el hallazgo de que entre los fallecidos haya menos concesiones de discapacidad puede ser debido a cualquiera de las siguientes causas: no solicitud de discapacidad, no concesión o fin del procedimiento administrativo debido al fallecimiento.

Los resultados confirman la información aportada por las trabajadoras sociales de las asociaciones federadas y colaboradoras de la FEPNC. Y, a pesar de no disponer de datos publicados con los que se pueda contrastar esta información, podemos considerar que los datos son válidos. La muestra presenta una distribución de tipos de cáncer similar a la de RETI-SEHOP (Peris Bonet et al., 2018) que aporta datos poblacionales y de la distribución y supervivencia en el primer año similar a la publicada en Europa (Gatta et al. 2014: 35). Aunque se pudiera considerar sesgada al no disponer de información de algunas comunidades autónomas (Baleares, Cantabria, Cataluña, Galicia, País Vasco) y escasez de casos de algunos de los tumores infantiles poco frecuentes (melanomas malignos y otras neoplasias epiteliales malignas), el volumen de casos estudiado (1288), la amplia representatividad de comunidades autónomas y tumores infantiles, así como el hecho de que es el único estudio en nuestro país en el que se recoge información de estas características, determinan la validez de los resultados obtenidos.

La escasez de solicitudes se puede deber, por una parte, a la actual normativa estatal y autonómica (la ley actual expresa que se solicite al finalizar el tratamiento) y por otra, según información aportada por las trabajadoras sociales de las diferentes asociaciones participantes, a la falta de información de las familias sobre la posibilidad de solicitarla o a motivos personales, entre los que se encuentra el temor de los padres a los efectos que pueda tener el que se etiquete a su hijo como persona con discapacidad. 
Sin embargo, la actual normativa estatal no parece ser un impedimento para que se solicite la discapacidad desde el inicio del diagnóstico y que se conceda en un plazo de tiempo admisible (1,2 meses). Hay comunidades autónomas en las que, sin una normativa autonómica que matice la estatal, se están solicitando valoraciones de discapacidad antes de los 6 meses y se están obteniendo resoluciones en un mes o menos. En las comunidades autónomas de Principado de Asturias, Canarias, Castilla y León y Comunidad de Madrid entre el $40 \%$ y el $57 \%$ de las solicitudes de discapacidad se están realizando al mes o menos. Y en las comunidades de Andalucía y Comunidad Valenciana en torno al $80 \%$ de las solicitudes se conceden en un mes o menos.

En coherencia con la percepción de las trabajadoras sociales se encuentra que el mayor porcentaje de concesiones de discapacidad son con un grado entre el $33 \%$ y el $65 \%(57,6 \%)$. Destaca la situación de la Comunidad Valenciana en la que se encuentra una asociación estadísticamente significativa con un grado de discapacidad $>=65 \%(p<0,05)$. Es una de las comunidades con un periodo más dilatado de solicitud de discapacidad desde el diagnóstico (el $44,3 \%$ de las solicitudes de discapacidad se realizan a partir de los 6 meses del diagnóstico) y con menor tiempo de resolución desde la solicitud (el $84,4 \%$ de las solicitudes se resuelven en un mes o menos). Este hecho es debido a que se está aplicando el criterio de agilización por el que se realizan las solicitudes de discapacidad a los 6 meses del diagnóstico; momento en el que una parte importante de niños continúan con tratamientos agresivos que suponen un alto porcentaje de valoración de discapacidad.

Aunque estos datos no pueden corroborar los de otros autores, al no existir estudios de referencia en España, cada vez más desde diferentes ámbitos sociales y políticos se admite que se han de homogeneizar los criterios de solicitud y resolución de discapacidad para niños con cáncer a nivel estatal (PNL 161/000290; PNL 161/000839; PNL 387/16; PNL 09/PNLP-00054; Gobierno de Canarias, 2017). Las diferencias territoriales determinan que muchas familias queden exentas de las ayudas que se conceden al disponer de un grado de discapacidad, al menos del $33 \%$, para contribuir a paliar los altos costes que desde el momento del diagnóstico suponen el hacer frente a esta enfermedad. Frecuentemente los padres de los niños y adolescentes con cáncer tienen que desplazarse de su lugar de origen a los hospitales de referencia, fuera de su provincia o comunidad autónoma, ya que de ese modo se posibilita un diagnóstico y unos tratamientos más apropiados.

La modificación del RD 1971/1999, de 23 de diciembre, indicando que a todos los niños y adolescentes con un diagnóstico de cáncer se les ha de valorar con un mínimo del $33 \%$ de discapacidad, como ya se ha hecho en el caso de personas con una coinfección de VIH y virus C de Hepatitis (RD 1169/2003, de 12 de septiembre), permitiría una aplicación homogénea por parte de todos los equipos de valoración de discapacidad, independientemente de la comunidad autónoma en la que se encuentren.

Añadido al cambio legislativo sería deseable, a su vez, aportar una mayor información a las familias sobre la posibilidad de solicitar la discapacidad desde el momento del diagnóstico, así como sensibilizar sobre los beneficios que les supondría el acceder a este tipo de ayuda.

Este trabajo es una primera aproximación al estudio de la discapacidad en niños y adolescentes con cáncer y a las diferencias de concesión de discapacidad entre comunidades autónomas. Para profundizar en el análisis de esta relación serían deseables nuevos estudios en los que se analicen, las posibles causas de estas variaciones entre comunidades autónomas para proponer medidas específicas que corrijan estas desigualdades territoriales. 
Referencias bibliográficas

Armstrong G, et al. (2014): "Aging and risk of Severe, Disabling, Life-Threatening and fatal events in the Chilhood Cancer Survivor study”. Journal of Clinical Oncology, 32 (12): 1218-1227.

Barrera, M. et al. (2005): "Educational and social late effects of childhood cancer and related clinical, personal, and familial characteristics”. Cancer, 104: 1751-1760.

España. Proposición no de ley, número de expediente 161/000290, sobre la valoración de la discapacidad de los menores con cáncer. Presentada por el grupo parlamentario socialista. Diario de sesiones del Congreso de los Diputados. Comisión para las políticas integrales de la discapacidad, 20 de diciembre de 2016, núm. 90, pp.13-16 (en línea). <http://www.congreso.es/public_oficiales/L12/CONG/DS/CO/DSCD-12-CO-90.PDF>, acceso 16 de abril de 2019.

España. Proposición no de ley, número de expediente 161/000839, sobre el reconocimiento del grado de discapacidad del $33 \%$ a los niños con cáncer. Presentada por el grupo parlamentario mixto. Diario de sesiones del Congreso de los Diputados. Comisión de sanidad y servicios sociales, 23 de febrero de 2017, núm. 128, pp.1721 (en línea). <http://www.congreso.es/public_oficiales/L12/CONG/DS/CO/DSCD-12-CO-128.PDF>, acceso 16 de abril de 2019.

España. Proposición no de ley, número de expediente 387/16, sobre menores afectados por cáncer (neoplasias) o enfermedades crónicas graves. Presentada por el grupo G.P. Ciudadanos-partido de la ciudadanía. Boletín Oficial de las Cortes de Aragón, 23 de noviembre de 2016, núm. 120, pp. 9296-9297 (en línea). <http://bases. cortesaragon.es/bases/boca2.nsf/(D)/154CB37A0DDF9FC4C12580740063804D?OpenDocument >, acceso 16 de abril de 2019.

España. Proposición no de ley, número de expediente 09/PNLP-00054, para el reconocimiento del $33 \%$ de discapacidad a los niños y niñas enfermas de cáncer. Presentada por el grupo parlamentario socialista. Boletín Oficial de Castilla-La Mancha, 28 de julio de 2017, núm. 140, pp. 5354-5355 (en línea). <https://www.cortesclm. es/web2/paginas/publicaciones/boletin/boletin9/pdf/140.pdf>, acceso 16 de abril de 2019.

España. Real Decreto 1971/1999, de 23 de diciembre, de procedimiento para el reconocimiento, declaración y calificación del grado de minusvalía. Boletín Oficial del Estado, 26 de enero de 2000, núm. 22, pp. 3317-3410 (en línea). <https://www.boe.es/buscar/pdf/2000/BOE-A-2000-1546-consolidado.pdf>, acceso 16 de abril de 2019.

España. Real Decreto 1856/2009, de 4 de diciembre, de procedimiento para el reconocimiento, declaración y calificación del grado de discapacidad, y por el que se modifica el Real Decreto 1971/1999, de 23 de diciembre. Boletín Oficial del Estado, 26 de diciembre de 2009, núm. 311, pp. 110413-110415 (en línea). <http://sid.usal. es/idocs/F3/LYN15025/3-15025.pdf>, acceso 16 de abril de 2019.

España. Real Decreto 1364/2012, de 27 de septiembre, por el que se modifica el Real Decreto 1971/1999, de 23 de diciembre, de procedimiento para el reconocimiento, declaración y calificación del grado de discapacidad. Boletín Oficial del Estado, 11 de octubre de 2012, núm. 245, pp. $72772-72774$ (en línea). <http://www.boe.es/ diario_boe/txt.php?id=BOE-A-2012-12686>, acceso 16 de abril de 2019.

España. Real Decreto Legislativo 1/2013, de 29 de noviembre, por el que se aprueba el Texto Refundido de la Ley General de derechos de las personas con discapacidad y de su inclusión social. Boletín Oficial del Estado, 3 
de diciembre de 2013, núm. 289, pp. 95635-95673 (en línea). <https://www.boe.es/buscar/doc.php?id=BOEA-2013-12632>, acceso 16 de abril de 2019.

España. Real Decreto Legislativo 1169/2003, de 12 de septiembre, por el que se modifica el anexo I del Real Decreto 1971/1999, de 23 de diciembre, de procedimiento para el reconocimiento, declaración y calificación del grado de minusvalía. Boletín Oficial del Estado, 4 de octubre 2003, núm. 238, pp. 36136-36138 (en línea). <https://www.boe.es/boe/dias/2003/10/04/pdfs/A36136-36138.pdf>, acceso 22 de abril de 2019.

Gatta, G. et al. (2014): "Childhood cancer survival in Europe 1999-2007: results of EUROCARE-5--a populationbased study". The Lancet Oncology. 15 (1): 35-47.

Gobierno de Canarias (17 de marzo de 2016): "Patricia Hernández dicta una resolución definitiva que unifica el grado de discapacidad para niños enfermos con cáncer de ambas provincias". Comunicado de prensa (en línea). <https://www.gobiernodecanarias.org/noticias/hemeroteca/epsv/70351/patricia-hernandez-dictaresolucion-definitiva-unifica-grado-discapacidad-ninos-enfermos-cancer-ambas-provincias>, acceso 16 de abril de 2019.

Hjern, A., et al. (2007): "Disability in adult survivors of childhood cancer: a Swedish national cohort study". Journal of Clinical Oncology, 25: 5262-5266.

Hudson, M. M. et al. (2013): "Clinical ascertainment of health outcomes among adults treated for childhood cancer”. JAMA, 309 (22): 2371-2381.

Oetfinger K, et al. (2006). "Chronic Health Conditions in Adult Survivors of Childhood Cancer". N England J Med, 355: 1572-1582.

Organización Mundial de la Salud (2001): Clasificación Internacional del Funcionamiento de la Discapacidad y de la Salud (CIF). Madrid: Ministerio de Trabajo y Asuntos Sociales (en línea). <http://www.imserso.es/InterPresent2/ groups/imserso/documents/binario/435cif.pdf>, acceso 16 de abril de 2019.

Peris Bonet, R. et al. (2018): Cáncer infantil en España. Estadísticas 1980-2017. Registro Español de Tumores Infantiles (RETI-SEHOP). Valencia: Universitat de València (ed. preliminar, CD-Rom). 